Meta

Journal des traducteurs

Translators' Journal

\title{
La problématique de la traduction du Coran : étude comparative de quatre traductions françaises de la sourate " La lumière "
}

\section{Chédia Trabelsi}

Volume 45, numéro 3, septembre 2000

La traduction dans le monde arabe

URI : https://id.erudit.org/iderudit/004504ar

DOI : https://doi.org/10.7202/004504ar

Aller au sommaire du numéro

Éditeur(s)

Les Presses de l'Université de Montréal

ISSN

0026-0452 (imprimé)

1492-1421 (numérique)

Découvrir la revue

Citer cet article

Trabelsi, C. (2000). La problématique de la traduction du Coran : étude comparative de quatre traductions françaises de la sourate "La lumière ". Meta, 45(3), 400-411. https://doi.org/10.7202/004504ar

\section{Résumé de l'article}

Quel que fût l'objectif des traductions françaises du Coran, celui-ci a toujours suscité l'intérêt des traducteurs. C'est que ce texte arabe se caractérise par une densité sémantique - une multitude, toujours ouverte, de lectures ou d'interprétations dont certaines sont accréditées par les grands exégètes musulmans - dont le traducteur doit prendre connaissance s'il ne veut pas risquer de donner une traduction qui réduirait ou occulterait les messages coraniques, d'où les différentes méthodes utilisées par les traducteurs pour combler au maximum les lacunes sémantiques. De plus, ce texte sacré se distingue par une richesse stylistique telle qu'il est considéré par les Arabes comme inimitable, ainsi se sont développées deux tendances parmi les traducteurs: certains optent pour une langue châtiée afin de s'approcher autant que possible de la richesse du style arabe. D'autres préfèrent imiter ce style afin d'essayer d'en refléter la beauté. Mais dans quelle mesure ces traductions françaises littérales peuvent-elles être fiables? 


\title{
La problématique de la traduction du Coran: étude comparative de quatre traductions françaises de la sourate «La lumière»
}

\author{
CHÉDIA TRABELSI \\ Université de Tunis I, Tunis, Tunisie
}

\begin{abstract}
RÉSUMÉ
Quel que fût l'objectif des traductions françaises du Coran, celui-ci a toujours suscité l'intérêt des traducteurs. C'est que ce texte arabe se caractérise par une densité sémantique - une multitude, toujours ouverte, de lectures ou d'interprétations dont certaines sont accréditées par les grands exégètes musulmans - dont le traducteur doit prendre connaissance s'il ne veut pas risquer de donner une traduction qui réduirait ou occulterait les messages coraniques, d'où les différentes méthodes utilisées par les traducteurs pour combler au maximum les lacunes sémantiques. De plus, ce texte sacré se distingue par une richesse stylistique telle qu'il est considéré par les Arabes comme inimitable, ainsi se sont développées deux tendances parmi les traducteurs: certains optent pour une langue châtiée afin de s'approcher autant que possible de la richesse du style arabe. D'autres préfèrent imiter ce style afin d'essayer d'en refléter la beauté. Mais dans quelle mesure ces traductions françaises littérales peuvent-elles être fiables?
\end{abstract}

\begin{abstract}
Whatever the objectives of the French translations of the Koran, the latter has always triggered an interest among translators. The Arabic text is characterised by a semantic denseness, an ever-open multitude of readings and interpretations - of which only some are accredited by the great Islamic exegetes - that the translator must be aware of if $\mathrm{s} /$ he does not want to take the risk of producing a translation that may obliterate part or all of the Koranic message. Hence the different methods used by translators in order to bridge semantic gaps as much as they can. Moreover, this canonical/Holy text is marked off by such a stylistic richness that Arabs deem it unique and inimitable. Hence the two tendencies among translators: a first category resorts to an intricate, refined French in order to re-create the elaborateness of the original Arabic style; a second category opts for an imitation of that style in order to mirror its beauty. To what extent can these literal French translations be reliable?
\end{abstract}

\section{MOTS-CLÉS/KEYWORDS}

Coran, traduction, sémantique, stylistique, Islam

\section{Introduction}

Le Coran, comme tous les textes sacrés, n'a pas laissé indifférents les lecteurs et les traducteurs dans toutes langues, qu'ils soient musulmans ou non, qu'ils soient monothéistes ou autres.

Il est vrai que l'intérêt suscité par le Coran, ainsi que les intentions des traducteurs, ont varié selon les communautés, les langues, les époques et les contextes politico-religieux d'un pays à l'autre ou d'une région à l'autre (Blachère 1991: 264277). Mais si nous prenons le cas de la France, par exemple, nous remarquons que 
c'est surtout à partir du XVII ${ }^{\mathrm{e}}$ siècle que ce livre sacré a commencé à susciter un vif intérêt chez une certaine classe: «la bonne société et Voltaire» (Blachère 1991: 270). Depuis, ces traductions françaises sont devenues de plus en plus nombreuses jusqu'à nos jours.

Le nombre de ces traductions «des sens du $\operatorname{Coran}^{1}$ » en français a, en effet, actuellement dépassé les cent vingt. Elles ont été publiées dans plusieurs pays et les traducteurs sont de différentes nationalités et religions. Les intentions de ces traducteurs sont également diverses : certains ont fait de leurs traductions un instrument de défiguration de l'Islam; d'autres, au contraire, en ont fait un moyen de propagande ou de découverte de cette religion par les non-musulmans; d'autres, enfin, considèrent leur traduction comme une contribution au patrimoine mondial, un enrichissement intellectuel de tout citoyen du monde, quelle que soit sa confession.

Par ailleurs, les méthodes adoptées par ces traducteurs sont multiples. Certains se contentent de traduire le Coran. D'autres accompagnent leurs traductions d'une étude introductive sur l'Islam, la vie de Mahomet, les conditions de la révélation du Coran, les multiples interprétations, exégèses ou sens possibles des chapitres (ou sourates) de ce livre et donnent ainsi une idée sur la difficulté de traduire le texte arabe sacré dans leur langue respective. Leur objectif est de préparer le lecteur non musulman et non arabophone à recevoir un texte qui non seulement n'est pas authentique - il n'est qu'une version humaine, créée d'un texte divin incréé ${ }^{2}$-, mais dont il est également impossible de cerner et de traduire tous les sens, quelles que soient les compétences de l'exégète et du traducteur.

Outre ce souci sémantique, les traducteurs du Coran n'ont pas suivi la même démarche linguistique. Certains, en effet, ont préféré être fidèles à la langue arabe de ce livre sacré et ont pris soin de l'annoncer dans leur introduction, et ce, afin de donner au lecteur français une idée sur le fond mais également, pensent-ils, sur la forme arabe sacrée. D'autres, tels Kazimirski et Mazigh dont nous allons voir plus loin des échantillons de traduction, ont privilégié la fidélité à la langue d'arrivée pour l'expression de ce fond. Il est évident que l'effet obtenu - et donc la qualité de ces traductions — n'est pas le même.

Toutefois, le Coran n'a fait l'objet de traductions françaises que relativement tard. En effet, un fort courant musulman traditionnel, se fondant, d'une part, sur l'impossibilité de cerner tous les sens du Coran, que seul Dieu connaît, et, d'autre part, sur l'inimitabilité de sa langue arabe, n'admettait pas la traduction de ce texte sacré. Ce n'est qu'au $\mathrm{xx}^{\mathrm{e}}$ s. surtout que les traducteurs musulmans sont passés outre ce courant et ont donné de ce livre sacré des versions en différentes langues, le Coran, à l'instar de la Bible, n'ayant pas seulement une portée communautaire mais une valeur œcuménique dont l'humanité est en droit d'apprendre le contenu.

Mais si ce courant traditionnel a perdu de son autorité, il n'a pas pour autant tout à fait disparu; encore se manifeste-t-il à travers des individus, ici et là, de temps à autre. En témoigne le livre arabe publié par le Tunisien Mahmoud Chabaâne (1984: 150) qui compare six traductions françaises du Coran — celles de Masson, de Kazimirski, de Mazigh, de Hamidullah, de Blachère et de Grojean — pour en conclure que la traduction de ce texte sacré est impossible. D'après lui, il suffit donc au traducteur d'en traduire les exégèses qui lui semblent les plus importantes, ou les plus plausibles, ou bien d'en écrire lui même une exégèse directement dans la langue étrangère de son choix! 
Si ce courant a vu le jour et qu'il a connu à un certain moment de la virulence, c'est parce que le Coran est un texte d'une ampleur sémantique et linguistique extraordinaire, de loin supérieure à celle de tout texte littéraire, quelle qu'en soit la teneur. C'est ce qui a fait que les adeptes de ce courant ont toujours considéré comme un affront à la parole divine toute tentative de traduction.

Cela n'a pas empêché les traducteurs d'essayer d'en donner une idée tout en étant convaincus - qu'ils soient musulmans ou autres, croyants ou non - que leur version ne peut être que très approximative comparée au texte arabe initial. Le problème essentiel demeure de nature sémantique; le volet stylistique n'en est pas moins important. Les quelques échantillons des quatre traductions que nous avons comparées le prouvent.

Nous avons, en effet, choisi deux versions françaises récentes faites par deux traducteurs tunisiens, Sadok Mazigh et Slaheddine Kechrid, ainsi que deux autres versions plus anciennes, celle de Régis Blachère et celle de Kazimirski.

\section{LA PROBLÉMATIQUE DE LA TRADUCTION DU TEXTE CORANIQUE}

La première tâche du traducteur est de cerner, au cours de ses lectures des différentes exégèses reconnues, accréditées du Coran, les interprétations les plus fortes, les plus fréquentes de chaque verset, de chaque sourate et de prendre connaissance également des conditions de la révélation de tous ces versets ${ }^{4}$. La raison en est la multitude des interprétations faites, ou encore possibles, de ce texte arabe sacré toujours ouvert à une foule de lectures de toutes tendances. Sans ce travail préalable et ardu, il est impossible de traduire un grand nombre de versets quelle que soit la compétence linguistique du traducteur dans les deux langues de départ et d'arrivée, car le Coran comprend des versets dont le sens est évident — ayat bayyinat — et d'autres dont le contenu est ambigu, équivoque - ayat moutachabihat. L'apport cognitif des exégèses coraniques est ainsi capital dans toute tentative de traduction. Cependant, il est des traducteurs qui, parce qu'ils n'ont pas suffisamment lu d'exégèses coraniques, ou parce qu'ils se sont contentés d'exégèses dites «faibles», c'est-à-dire non fréquentes, non accréditées par la plupart des grands exégètes, ou encore parce qu'ils n'ont pas la compétence linguistique arabe ou française requise, ont fait des traductions comportant des erreurs d'interprétation de deux sortes: des erreurs personnelles et des erreurs d'ordre général.

\section{Les erreurs personnelles du traducteur}

Nous avons relevé toutes sortes d'erreurs de ce genre chez les quatre traducteurs mentionnés même si leur fréquence et leur nature varient d'un traducteur à l'autre. La sourate (ou chapitre) que nous avons prise comme échantillon est la vingtquatrième: «La lumière», révélée à Médine et comprenant 64 versets (V) ou sousparties. Ces erreurs dues au seul traducteur sont de divers types; nous en sélectionnons quelques-unes: 


\section{a) erreur par rétrécissement du sens accrédité:}

V 38: /wallahou yarzoukou man yachaou bighayri hisabin/

/yarzoukou/ est un verbe qui signifie «accorder à une créature tous types de biens moraux ou matériels»

Kazimirski (Ka) le traduit ainsi : «Dieu donne la nourriture à qui il veut »

Blachère $(\mathrm{B})$, Mazigh $(\mathrm{M})$ et Kechrid $(\mathrm{K})$ le traduisent respectivement par «donner attribution à », «dispenser ses dons à » et «donner ses biens à».

\section{b) erreur par omission:}

V 4: /walladhina yarmouna al mohsanati thomma lam yatou biarbaati chouhada fajlidouhom thamanina jaldatan/

/jalada/ est un verbe qui signifie «donner des coups de fouet». C'est ce que mentionnent clairement $\mathrm{Ka}, \mathrm{B}$ et $\mathrm{M}$, mais $\mathrm{K}$ se contente de traduire par «Ceux qui jettent une accusation d'adultère aux femmes chastes, donnez-leur quatre-vingts coups ».

\section{c) erreur par manque de clarté du sens exprimé:}

/mohsanatou/ est un terme arabe qui désigne les femmes chastes et mariées. Tel quel, c'est-à-dire sans traduction ou sans explication, il ne peut être compris par un lecteur ignorant la langue arabe. Toutefois, B l'a laissé tel quel et sans même ajouter une note ou l'accompagner du terme français:

V 23: /inna alladhina yarmouna al muhsanati al ghafilati al mouminati louinou fiddounya wal a khira/:

$\mathrm{B}$ : «Que ceux qui visent [en leur honneur] les muhsana insoucieuses [mais] croyantes soient maudits en la [vie] immédiate et dernière.»

$\mathrm{M}, \mathrm{Ka}$ et $\mathrm{K}$ ont traduit respectivement par «femmes [...] fortes de leur vertu», «femmes vertueuses» et «femmes chastes».

d) erreur par ambiguïté du sens exprimé:

V 31: /waqol lilmouminati... la yobdina zinatahonna illa liboulatihinna aw... abai boulatihinna/

$M$ traduit ce verset comme suit:

«Dis de même aux croyantes qu'elles veillent à ne pas étaler leurs ornements sauf devant leurs époux... leurs beaux-pères...»

L'expression arabe /abai boulatihinna/ est sans aucune ambiguïté. Elle ne signifie que «Les pères de leurs maris». Or, la traduction par «beaux-pères", même en contexte, n'est pas claire. Que doit comprendre, en effet, le lecteur français: la femme peut étaler ses ornements devant le père de son mari, ou bien devant le mari de sa mère? «Beaupère» ne désigne-t-il pas, en effet, ces deux hommes à la fois en français?

Pour éviter cette ambiguïté, Ka a traduit par «père de leurs maris», B par «à leurs époux, ou à leurs pères» - le contexte de sa traduction montre clairement qu'il s'agit des pères des maris - et $\mathrm{K}$ par «le père de leur mari».

\section{Les erreurs d'ordre général}

Ce ne sont pas en réalité des erreurs: ce sont plutôt des interprétations différentes des mêmes versets faites par un même exégète, ou bien par plusieurs exégètes et transmises par les traducteurs. Le résultat en est que les mêmes versets ont des contenus partiellement différents d'un traducteur à l'autre.

L'exemple le plus remarquable que nous ayons noté dans cette sourate est celui du verset suivant: 
V 53: /wa aksamu billahi jahda aymanihim lain amartom layakhroujanna qol la toksimou taaton maaroufaton inna allaha khabiron bima taamaloun/

Deux traducteurs, $\mathrm{M}$ et $\mathrm{Ka}$, ont donné le même contenu de ce verset, à savoir :

$\mathrm{M}$ : «Ils jurent par Dieu leurs grands serments que si jamais tu leur en donnes l'ordre ils voleront aussitôt au combat. Dis-leur: "Gardez vos serments! Ce qui compte, c'est d'obéir. Dieu observe toutes vos actions».

$\mathrm{Ka}$ : «Ils ont juré, par le nom de Dieu, le plus solennel des serments, que si tu leur ordonnais de marcher au combat ils le feraient. Dis-leur: Ne jurez point; c'est l'obéissance qui a un prix. Dieu connaît vos actions».

La traduction de B reste plus vague; c'est l'expression quasi littérale de l'arabe:

B: "[Ces gens] ont juré par Allah, en des serments solennels, que si tu leur en donnes certes l'ordre, ils se mettront [en campagne] [Prophète!] dis[-leur] ...Ne jurez point! Obéissance reconnue [convenable]! Allah est bien informé de ce que vous faites».

Par contre, K transmet un sens clairement différent:

$\mathrm{K}$ : «Ils jurèrent de toute la force de leurs serments: "Si tu le leur ordonnais, ils sortiraient à coup sûr. Dis: 'Ne jurez donc pas! Votre façon (verbale) d'obéir est bien connue. Dieu est parfaitement au courant de ce que vous faites'” ".

\section{Les compléments d'information}

Le fait que le Coran se prête à plus d'une lecture et qu'il a été révélé dans diverses conditions a poussé les traducteurs à donner des compléments d'information sous différentes formes au lecteur non arabophone et/ou non musulman afin de l'aider à mieux comprendre. On sait d'ailleurs que même le lecteur arabe et musulman a besoin de ces compléments cognitifs - mais peut-être pas toujours aux mêmes endroits ni de même nature — pour que le texte coranique lui soit plus clair.

Si nous prenons le cas de Mazigh, qui a publié sa traduction en deux volumes, nous constatons qu'il regroupe toutes ses notes - dont la longueur varie entre une demi-page et une page et demie environ - entre la traduction de tout le Coran et le texte arabe original. Ces notes sont le plus souvent des commentaires sur tel ou tel fait mentionné dans telle ou telle sourate. Elles peuvent également être des éclaircissements portant sur les conditions de la révélation d'une sourate particulière ou de quelques versets de cette sourate. Ces notes peuvent encore compléter ou expliquer les ellipses et/ou les allusions faites à un événement ou à une personne donnée dont il est question dans une certaine sourate. Par exemple, dans les quatre traductions qui nous concernent, les traducteurs expliquent, plus ou moins brièvement, la raison de la révélation des seize premiers versets de «La lumière »: l'accusation d'adultère lancée à Aïcha, l'épouse préférée du Prophète, par un groupe de musulmans dont comble d'infamie - certains, indigents, étaient aidés financièrement par le père de Aïcha, le Khalife Abou Bakr.

Quant à Kazimirski et à Kechrid, ils accompagnent leur traduction de notes en bas de page pour les mêmes raisons que Mazigh, mais ces notes sont plus courtes et plus rares chez Kazimirski - quatre en tout pour la sourate qui nous intéresse et vingt chez Kechrid pour le même chapitre. De plus, les notes de ce dernier traducteur - dont la longueur varie entre une et vingt lignes - sont parfois des commentaires personnels des réalités musulmanes actuelles (ex.: note 1, p. 462). Elles peuvent être 
également des propositions d'interprétations différentes mais de contenus proches (ex. : note 2, p. 469).

Blachère, quant à lui, adopte une méthode qui diffère beaucoup des autres traducteurs. Il reclasse les sourates du Coran selon la chronologie de leur révélation. Toutefois, ce reclassement n'est pas toujours possible parce qu'il y a des versets dont les spécialistes ne sont pas sûrs de la date de révélation. De plus, il donne en avantpropos une bibliographie, une explication du titre du chapitre ainsi qu'un aperçu historique et thématique de la sourate afin d'éclairer davantage le lecteur français non musulman à qui est destinée cette traduction. En outre, il divise chaque sourate selon les sujets dont elle traite et donne un titre à chacune de ces parties. D'autre part, comme il a publié une introduction au Coran — séparée de la traduction de ce livre - où il traite de la spécificité essentielle de ce texte sacré, à savoir la multitude des interprétations possibles d'un grand nombre de versets, il donne souvent, au sein même du texte traduit, deux ou trois versions reconnues - séparées par des barres et désignées par les lettres A, B et C — du même verset (exemple: V 36, p. 1013-1014 de «La lumière»). Par ailleurs, il enrichit chaque sourate en bas de page par de nombreuses notes dont certaines sont très longues (exemple: note 35, p. 1012-1013; elle prend plus d'une page en caractères fins). Le contenu de ces notes est variable: en plus du même contenu que les notes des autres traducteurs, Blachère établit des comparaisons sur certains points avec la Bible ou avec la poésie arabe pré-islamique (exemple: la longue note 35, citée plus haut, où il commente la principale parabole de cette sourate: "Allah est la lumière des cieux et de la terre... », laquelle parabole a donné son nom à ce chapitre.

\section{LES SPÉCIFICITÉS STYLISTIQUES}

Outre ces types de compléments d'information coranique, les quatre traducteurs ont adopté d'autres méthodes soit pour compléter ce qui manque à la compréhension du sens d'un verset, soit pour rendre le verset plus transparent. Ces ajouts, parfois obligatoires, parfois facultatifs, relèvent du sémantique et du stylistique à la fois comme nous allons le voir dans les exemples pris dans les versets 10, 20 et 40 .

\section{Les ajouts ou compléments cognitifs obligatoires}

V 10: /wa lawla fadhlou llahi alaykoum wa rahmatouhou wa anna allaha tawwabon hakim/

V 20: /wa lawla fadhlou allahi alaykom warahmatouhou wa anna allaha raoufon rahim/

/lawla/ est un outil qui exprime la supposition — l'équivalent de «si» — dont l'emploi nécessite deux propositions: une principale et une subordonnée. La seconde constitue une réponse à la première. Toutefois, il est un style elliptique classique et courant dans le Coran où seule la proposition principale est explicite et la réponse est implicite. Cette tournure en suspens ou cette forme d'ellipse n'est pas française et, si elle reste telle quelle dans cette langue, le contenu reste incompris. C'est pourtant le style qu'adopte Kechrid dans sa traduction. Cela n'est nullement étonnant de sa part puisqu'il annonce dans son introduction au Coran que son objectif est de rester fidèle au style du texte arabe afin de donner une idée du style coranique au lecteur non arabophone. Aussi accompagne-t-il le verset 10 ainsi traduit: 
«Sans la générosité que vous devez à Dieu en même temps que sa miséricorde et n'était

la facilité de Dieu à accepter votre repentir ainsi que sa sagesse...»

Note: "Sous-entendu: "Qu'adviendrait-il de vous?" ».

Il procède de la même manière pour la traduction du verset 20 :

«N'étaient la générosité que vous devez à Dieu ainsi que sa miséricorde et si Dieu n'était pas plein de compassion et de clémence...»

Note: «Sous-entendu: "Vous auriez subi des tourments énormes"».

Quant à Blachère, dont le style de la traduction est extrêmement fidèle au style arabe, voire même plus littéral que celui de Kechrid, il adopte la même démarche que ce dernier : il complète l'information arabe implicite du verset 10 en bas de page, et il fait un rappel du même trait en bas de la page où il traduit le verset 20 que voici:

V 20: «N'eussent été la faveur d'Allah envers vous et sa miséricorde et [n'eût été] qu'Allah est indulgent et miséricordieux.»

Note: «20: Même trait vt.10; V. la note» (Blachère 1950: 1007)

Mais ce qui est frappant chez ces deux traducteurs, c'est qu'aucun d'entre eux, même s'il arrive qu'ils fassent des commentaires sur tel ou tel trait linguistique du Coran, n'a expliqué au lecteur français, dans les notes de cette sourate, cette spécificité stylistique arabe malgré sa fréquence dans le Coran!

Quant à Kazimirski et à Mazigh, les deux traducteurs qui ne sont pas restés fidèles au style arabe mais qui se sont exprimés dans un style français classique pour l'un — rappelons que Kazimirski a publié sa traduction pour la première fois en 1820 et contemporain pour l'autre, ils ont adopté une méthode différente pour expliciter l'information implicite. En effet, au lieu d'ajouter des notes de bas de page, ils ont intégré l'information manquante dans la traduction de sorte que le lecteur français ne puisse se douter un instant de la tournure utilisée en arabe et rendue ainsi:

V 10:

- Ka: «Si ce n'était la grâce inépuisable de Dieu et sa miséricorde, il vous punirait à l'instant; mais il aime à pardonner, et il est miséricordieux.»

- $\mathrm{M}$ : «Ces dispositions témoignent, en vérité, de la grâce manifeste et de la bonté du Seigneur envers vous. Dieu est si enclin au pardon, d'une si indulgente sagesse.»

V 20:

- Ka: «Si ce n'était la grâce inépuisable de Dieu et sa miséricorde, il vous punirait; mais il est humain et miséricordieux. »

- M: «N'eût été la grâce de Dieu envers vous et sa miséricorde, vous auriez déjà subi ses rigueurs. Mais Dieu est si bon, si compatissant.»

\section{Les ajouts d'information facultatifs}

Outre ces ajouts d'information obligatoires dont le lecteur français a besoin pour saisir le message intégral, les traducteurs ont employé une autre méthode pour que le contenu des versets soit plus transparent, comme le montre le verset suivant:

V 50: /afi kouloubihim maradhon am irtabou.../

Parmi ces quatre traducteurs, il n'y a que Mazigh qui ait essayé de rendre le sens de ce verset plus clair en optant pour une traduction légèrement explicative. En effet, il a 
ajouté les termes «profond» et «authenticité du Message», dont le sens est implicite dans le texte arabe:

"Leur cœur serait-il le siège d'un mal profond? Douteraient-ils de l'authenticité $d u$ Message? [...]»

Ce genre d'ajouts facultatifs visant à rendre les sens des versets plus accessibles à la compréhension du lecteur est d'ailleurs un trait caractéristique de la traduction de Mazigh, non seulement de la sourate «La lumière» mais aussi de tout le Coran.

Sur ce plan, la traduction de Kazimirski vient en second lieu. Quant à Kechrid et Blachère, ils donnent moins d'explications au sein même du texte traduit, étant donné que leur style est proche de l'arabe, en particulier celui de Blachère. Le style de ce dernier frappe en effet par sa ressemblance au texte original au point de donner des traductions dont le sens serait quasi incompréhensible pour un lecteur français, ou même pour un lecteur musulman ignorant l'arabe, s'il n'était accompagné d'une note explicative en bas de page. Exemple:

V 64 : /la tajalou douaa arrasouli baynakom ka douai baadhikom baadhan/

B : «Ne faites point de l'appel de l'Apôtre [lancé] parmi vous, comme [vous faites] de votre appel réciproque. $»^{5}$

La note explicative qu'il ajoute en bas de page (p. 1021-1022 et suivantes):

63. Du'a r-rasuli, «de l'appel de l'Apôtre». Les commt. glosent par nidâ'a, «apostrophe». Il peut s'agir aussi bien d'une convocation que d'une invitation; le sens semble donc être: Ne considérez pas que l'appel ou la convocation du Prophète ne mérite pas plus d'égards que l'appel réciproque que vous vous lancez. Autre sens: N'usez pas pour appeler le Prophète, des apostrophes dont vous vous servez entre vous.

\section{La littéralité ou la fidélité à l'arabe}

\section{a. La fidélité à l'expression et au terme}

Ce côté littéral, frappant chez Blachère et également apparent chez Kechrid, n'empêche pas toujours pour autant la compréhension des sens des versets. Toutefois, il dénature, à notre avis, la langue d'arrivée, qui en devient curieuse, et l'effet obtenu devient étrange.

En voici un exemple de Blachère:

V 16: /wa lawla idh samitoumouhou qoltom ma yakounou lana an natakallama bihadha sobhanaka hadha bohtanon adhim/

B: «Quand vous avez entendu [cette calomnie] que n'avez-vous dit: "Il n'est point de nous de parler de cela [Seigneur!], gloire à Toi! C'est une immense infamie (buhtan)!"”

Ainsi, l'expression française soulignée est presque une copie littérale de l'expression arabe transcrite et également soulignée. De plus, on constate que le traducteur tient à être fidèle à la langue arabe à un point tel qu'il accompagne le terme «infamie » de son équivalent arabe mis entre parenthèses (buhtan) mais qui n'est aucunement utile pour un lecteur français ignorant cette langue. Il suit d'ailleurs la même démarche dans la traduction des versets 11 (Kasaba), 38 et 55-56 (Zakat), 44 (dâbba), 60 
(haraj') et 63 (fitna) sans oublier les versets où il garde le terme aya - qui signifie, entre autres, «verset» — écrit en italique sans traduction ni note de bas de page (V 1 et $\mathrm{V}$ 57-58).

\section{b. La fidélité au temps}

Quant à Kechrid, sa fidélité à la langue arabe se situe certes au niveau de l'expression comme c'est le cas pour Blachère mais aussi - et contrairement aux autres traducteurs - au niveau de l'emploi des temps. Il n'y respecte pas en effet la spécificité de la langue française, langue d'arrivée, mais celle de la langue de départ, même s'il mentionne cette démarche clairement dans son introduction au Coran. C'est ce qui, à notre avis, dénature le texte traduit. En voici un exemple:

V 27: Iya ayyouhal ladhina amanou la tadkhoulou bouyoutan ghayra bouyoutikom hatta $[\ldots] /$

$\mathrm{K}$ : «Ô vous qui avez cru! N'entrez pas dans des maisons autres que les vôtres avant de [...].»

Ce qui frappe dans cette traduction est l'emploi du passé composé qui traduit un verbe arabe conjugué également au passé, /Fiil madhi/. Toutefois, la conjugaison du verbe /amana/ ( «croire») au passé composé, dans ce contexte bien particulier, fausse le sens exprimé en arabe. Il donne, en effet, l'impression que Dieu s'adresse à des gens qui ont cru en lui dans un temps passé mais qui ont perdu leur foi, alors que le verbe arabe exprime un passé apparent et un temps en réalité absolu. Il englobe aussi bien le passé que le présent et le futur, c'est-à-dire qu'il exprime la foi acquise depuis un certain temps passé et pour toujours, jusqu'à l'éternité. C'est ce qui a été clairement exprimé par l'emploi du présent dans les traductions de Blachère et de Mazigh qui ont conjugué le verbe «croire» au présent dans l'expression suivante pour lui donner cette valeur absolue: «Ô vous qui croyez!» Quant à Kazimirski, il a exprimé cette même valeur en employant un nom: "Ô croyants!»

La même remarque est d'ailleurs valable pour la traduction du verset 39 /walladhina Kafarou/ que seul Kechrid a rendu par l'emploi d'un verbe au passé composé — «Et ceux qui ont mécru» — alors que Blachère l'a traduit par un présent — «Les actions de ceux [au contraire] qui sont infidèles» — et Kazimirski et Mazigh l'ont exprimé respectivement par un nom — «Pour les incrédules» et «Quant aux mécréants ».

\section{Le respect des spécificités linguistiques françaises}

Néanmoins, si les traductions de Blachère et de Kechrid se caractérisent stylistiquement par cet aspect littéral, celles de Kazimirski et de Mazigh en particulier ont pour souci le respect des spécificités de la langue cible, le français. Même si un siècle et demi environ sépare les deux traductions, chacune revêt le style de son époque.

En effet, pour le lecteur français actuel, le texte de Kazimirski est rédigé dans un français classique qui rappelle plus un texte littéraire actuel extrêmement châtié qu'un texte français courant. Par contre, la traduction de Mazigh, datant d'environ vingt ans seulement, est rédigée dans un français moderne et soutenu.

Par ailleurs, si nous regardons de près, toujours du point de vue stylistique, les traductions de Mazigh et de Kazimirski, la première semble plus soignée essentielle- 
ment en ce qui concerne la variation des termes et des expressions ainsi qu'en ce qui a trait aux charnières.

\section{a. Les termes}

Sur le plan des termes, l'exemple de /alim/ est très significatif. Il a été utilisé dix fois dans la sourate et Mazigh lui a trouvé huit traductions différentes, c'est-à-dire qu'il n'a fait qu'une seule répétition. Kechrid, quant à lui, a trouvé sept traductions de ce terme, alors que Kazimirski n'en a trouvé que cinq. Mais ce qui est frappant, c'est que Blachère n'a donné qu'une seule traduction, qu'il a répétée huit fois: "omniscient». Est-ce encore par fidélité à l'arabe qu'il a conservé le même terme? Par ailleurs, Mazigh, Kazimirski et Kechrid ont respectivement traduit /alim/ aussi bien par les termes «omniscient», «savant» et «sachant» que par des expressions différentes.

\section{b. Les expressions}

Au niveau de l'expression, les deux traducteurs les plus fidèles à la langue de départ - Blachère et Kechrid - sont ceux qui ont donné une seule traduction de l'expression /hatta yoghnihim allahou min fadhlihi/, qui figure dans les versets 32 et 33 . Pour Blachère, "Allah les fera se suffire par sa faveur»; pour Kechrid, "Dieu les enrichira de sa générosité». Par contre, Mazigh et Kazimirski, soucieux d'éviter la répétition à laquelle répugne généralement la langue française, ont donné chacun deux traductions différentes de cette expression.

\section{c. Les charnières}

Pour ce qui est des charnières enfin, c'est Mazigh qui, beaucoup plus que les autres traducteurs, a introduit des charnières explicites là où elles ne sont qu'implicites en arabe. Il a également varié les charnières qui sont répétées en arabe. Son style a ainsi pris un aspect plus français, ses phrases étant articulées selon le rythme et les règles de cette langue et non selon ceux de la langue de départ, l'arabe. En voici un exemple pris de deux versets qui se suivent:

V 30: /qol lil mouminina yaghodhou min absarihim wa yahfadhou fouroujahom/

V 31: /waqol lil mouminati yaghdhodhna min absarihinna wa yahfadhna fouroujahonna/

V 30: «Dis aux croyants de tenir leurs yeux pudiquement baissés.»

V 31: «Dis de même aux croyantes de baisser non moins pudiquement leurs regards.»

Si nous comparons les trois autres traductions à celle-ci, nous constatons que celles de Blachère et de Kazimirski ne comprennent aucune charnière: elles sont deux propositions indépendantes qui se suivent et qui sont numérotées. Kechrid, quant à lui, emploie dans sa traduction une conjonction de coordination « et » pour coordonner les deux versets comme c'est le cas en arabe avec la conjonction /wa/.

\section{Conclusion}

Ce sont des échantillons qui sont loin d'être très représentatifs des spécificités stylistiques de chacune de ces quatre traductions. Les critères de fidélité en traduction diffèrent, d'après ces quelques échantillons, d'un traducteur à l'autre selon qu'ils 
optent pour la fidélité à la langue de départ comme Kechrid et Blachère en particulier, ou pour la fidélité à la langue d'arrivée comme Kazimirski et Mazigh. L'effet produit sur le lecteur diffère, bien entendu, d'une traduction à l'autre, selon la clarté du contenu qu'elle transmet et selon le style qu'elle adopte, lequel est évidemment, différent du style du texte original. Toutefois, et sans prétendre à la hauteur stylistique du Coran, réputé inimitable (/moôjiz/) certaines traductions, celle de Mazigh en particulier, ont atteint un niveau d'élaboration stylistique et une clarté sémantique tels qu'elles ont été classées parmi les meilleures versions françaises au monde.

Néanmoins, et malgré cette inimitabilité du Coran, ces versions françaises - les meilleures, bien entendu — ont le mérite de l'accessibilité de la langue et de la clarté des contenus qu'elles transmettent. En effet, outre la multitude des interprétations accréditées dont le lecteur doit prendre connaissance pour bien comprendre le texte coranique, la langue arabe du Coran est ressentie actuellement comme une langue très classique, peu courante et qui nécessite, par conséquent, que le lecteur arabe maîtrise bien sa langue pour bien pénétrer ce texte.

Par contre, il n'est pas nécessaire, à notre avis, que le lecteur français ait la même maîtrise de sa langue pour lire le Coran traduit. Il lui suffit d'en avoir un assez bon niveau pour saisir ce texte. La raison en est évidemment que les traductions françaises actuelles sont rédigées en français contemporain et non pas en un français classique, peu accessible.

Nous avons d'ailleurs constaté que ceux qui ne maîtrisent suffisamment pas l'arabe pour pouvoir lire le Coran ne se le font pas expliquer en arabe par un spécialiste mais y accèdent directement à l'aide d'une traduction française.

Cela soulève, à l'évidence, la question de la qualité, de la fiabilité ou de la fidélité d'une traduction. Certes, la fidélité est un critère complexe et nous en avons déjà vu une première tendance d'une part avec les traductions - littérales - de Blachère, surtout, et de Kechrid, et, d'autre part, une seconde avec celles — non littérales — de Kazimirski et de Mazigh. Notre préférence est claire.

Une traduction fidèle est à notre avis un texte qui, loin de négliger dans la langue d'arrivée le côté stylistique visant à se rapprocher de l'effet produit par le style équivalent du texte original, exprime clairement le même message transmis par ce dernier texte afin qu'il soit bien saisi par le lecteur de la traduction, d'où nos réserves à l'égard d'une traduction littérale qui, outre le fait qu'elle est stylistiquement lourde, voire parfois étrange, risque d'occulter partiellement ou complètement le message transmis et d'empêcher ainsi le lecteur de le décoder. Ce décodage ne serait possible dans le cas du Coran qu'à l'aide de notes explicatives du traducteur qui «sauveraient» sémantiquement la traduction. Mais, stylistiquement, celle-ci laisserait toujours à désirer. À quoi servirait-il donc d'imiter le style du texte original ? L'imitation, par la littéralité, donnerait-elle une idée de la beauté du texte coranique? Bien au contraire!

\section{NOTES}

1. En Islam, on parle de la traduction «des sens du Coran» et non de celle du Coran.

2. Tels Blachère et Kechrid dont nous allons voir, plus bas, la traduction.

3. Il se félicite, à ce propos, de la traduction française de quelques volumes de l'œuvre de Tabari, grand exégète musulman.

4. Afin d'être à même de comparer sémantiquement ces quatre traductions, nous avons nous-mêmes 
eu recours à deux exégèses accréditées: l'une classique d'El Baydhaoui, l'autre beaucoup plus récente du Tunisien Tahar Ben Achour.

5. Ce qui ne constitue pas un problème mais une richesse sémantique de ce texte sacré. Cette lecture plurielle est d'ailleurs, pour beaucoup de spécialistes, à porter au crédit de ce texte jamais clos, jamais statique, mais toujours ouvert à des interprétations nouvelles, toujours accessible à l'exégèse en tout lieu et en tout temps.

\section{RÉFÉRENCES}

BLAChÈRe, R. (1950): Le Coran: traduction selon un essai de reclassement des sourates, vol. 1, Paris, Librairie Orientaliste et Américaine, G. P. Maisonneuve \& Cie.

- (1991): Introduction au Coran, $2^{\mathrm{e}}$ éd., Paris, Maisonneuve et Larose.

Chabanne, M. (1984): Aïna Min al Koraan tarajimou al Koraan, Tunis, tabatou al qawmiyya linnachr.

KAZimirski (1970): Le Coran, Paris, Flammarion.

Kechrid, S. (1990): Le Coran, $5^{e}$ éd., Beyrouth, Dar El Gharb Al Islami.

Mazıgh, S. (1979) : Le Coran, Tunis, Maison Tunisienne d’Édition. 